\title{
Workload-aware Electromigration Analysis in Emerging Spintronic Memory Arrays
}

\author{
Sarath Mohanachandran Nair ${ }^{\dagger}$, Mehdi B. Tahoori ${ }^{\dagger}$, Manu Perumkunnil ${ }^{\ddagger}$, Houman Zahedmanesh ${ }^{\ddagger}$, Kristof Croes $^{\ddagger}$, \\ Kevin Garello ${ }^{\ddagger}$, Tommaso Marinelli*§ , Timon Evenblij ${ }^{\ddagger}$, Gouri Sankar Kar ${ }^{\ddagger}$, and Francky Catthoor ${ }^{\ddagger \S}$ \\ ${ }^{\dagger}$ Karlsruhe Institute of Technology, Germany \\ ${ }_{\ddagger}^{\ddagger}$ Imec vzw, Leuven, Belgium \\ $\S$ Katholieke Universiteit Leuven, ESAT, Belgium \\ ${ }^{*}$ Complutense University of Madrid, Spain
}

\begin{abstract}
Electromigration (EM) has emerged as a major reliability concern for interconnects in advanced technology nodes. Most of the existing EM analysis works focus on the power lines. There exists a limited amount of work which analyzes EM failures in the signal lines. However, various emerging spintronicbased memory technologies such as the Spin Transfer Torque Magnetic Random Access Memory (STT-MRAM) and the Spin Orbit Torque Magnetic Random Access Memory (SOT-MRAM) have high current densities as compared to the conventional Static Random Access Memory (SRAM). These high current densities can lead to EM failures in the signal lines such as bit-line (BL) of these memories. Furthermore, these signal lines have workloaddependent stress as opposed to the conventional DC stress of power distribution networks. In this work, we model the EM failures in the BL of a typical STT memory array with realistic workloads. The analysis is based on physics-based EM model, which is calibrated based on industrial measurement data. The results show that the current densities in the STT arrays can be large enough to cause EM failures in the signal lines with running realistic workloads and that these failures are highly workload-dependent.
\end{abstract}

Keywords-electromigration, spintronic memory, reliability

\section{INTRODUCTION}

Aggressive technology scaling has resulted in several reliability challenges for VLSI interconnects manufactured in advanced technology nodes. Among these, electromigration $(\mathrm{EM})$ is one of the major issues affecting back-end-of-line (BEOL) interconnects [1-3]. EM occurs due to the migration of metal atoms by the transfer of momentum between conducting electrons and atoms, resulting in the formation of a void at the cathode and a hillock at the anode end of the metal line. EM is typically divided into two phases, the nucleation phase and the growth phase. The nucleation phase is characterized by a void formation at the cathode end of the line. In the growth phase, the void grows and covers the cross-section of the line. As technology scales down, the current densities increase resulting in more metals atoms getting displaced, thus making the interconnects more susceptible to EM failures.

The emerging spintronic-based non-volatile magnetic random access memories (MRAMs) are seen as a viable solution to overcome the scaling challenges of conventional CMOS memories. Among the emerging MRAMs, the Spin Transfer Torque (STT) and the Spin Orbit Torque (SOT) MRAMs are the most promising technologies due to their several advantages such as high density, high endurance, low access latency, CMOS compatibility and immunity to radiation-induced soft errors [4, 5]. However, the comparatively high read and write currents still remain a challenge for this emerging memory technology. In particular, these high currents can aggravate the EM issues in the interconnects and negatively impact the reliability.

Most of the existing works on EM analysis for interconnects focus on the power grid network [6-8]. This is because the power/ground lines typically carry higher currents and hence have higher current densities as compared to signal lines. Furthermore, the current in the power/ground lines are unidirectional whereas the current in the signal lines are typically bi-directional. The bi-directional current in the signal lines lead to self-healing or EM recovery effects, which can drastically improve the life time of the wires. Hence the power/ground lines are more susceptible to EM failures since they not only have higher current densities, but also do not benefit from EM recovery effects.

However, it has been shown that even signal lines in Static Random Access Memory (SRAM) can have EM failures [9. 10]. These works focus on EM analysis for the read operation in SRAM, since the read current is unidirectional and hence self-healing effect is smaller. Here, the authors do not consider EM failures in the write operation since the average write current is assumed to be zero. This assumption is valid only for uniform workloads. However, for typical memory accesses, the workload is non-uniform, leading to a net average write current, which can result in EM failures. This is especially true for emerging spintronic memory technologies which have higher read and write currents as compared to SRAM. In addition, these memories have asymmetric write ' 0 ' and write ' 1 ' currents, which means that the average write current cannot be assumed to be zero. Hence it is important to investigate the possibility of EM failures in this emerging memory technology. Furthermore, the combination of workload-dependent current densities and asymmetric write makes the EM analysis in MRAM more challenging as compared to conventional SRAM.

In this work, we investigate the EM failures in the bit- 


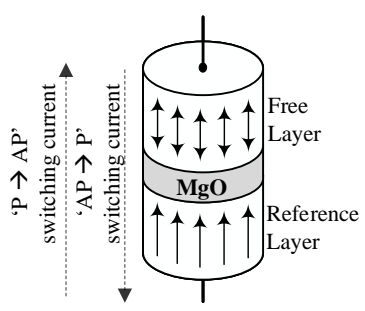

(a) MTJ

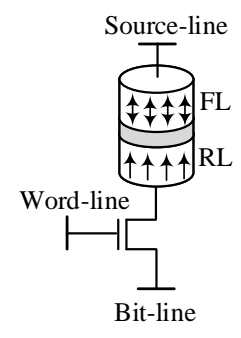

(b) Bit-cell
Fig. 1: STT-MRAM storing device

line (BL) of emerging spintronic memories, considering both the read and the write operations. As a representative illustration, our case study is based on STT-MRAM targeted at embedded cache/scratchpad applications, where the number of write cycles is up to $10^{15}$. The EM analysis considers realistic workloads from SPEC benchmarks which have been executed on an extended gem5-based simulation framework. Such workload-based analysis has been ignored in the previous studies. In addition, the existing EM modeling in signal lines for SRAM [9, 10] is based on simple Blech length formulation. In contrast, in this work, we perform EM analysis using the physics-based EM model developed in our previous works [11, 12], which is calibrated based on industrial measurement data. This model has been scaled to match the dimensions and material parameters of the BL of the STT array. Using this model, the mean-time-to-failure (MTTF) due to EM of different bit-lines in a typical STT array configuration is computed for different workloads.

The results show that some of the bit-lines are immortal to EM effects, while others are highly susceptible to EM. This difference in MTTFs of different bit-lines is due to their highly different workload-dependent current densities. This work highlights the importance of incorporating proper EM mitigation techniques for this emerging memory technology to ensure reliable operation.

The rest of the paper is organized as follows. Section II explains the basics of spintronic memory technology as well as the EM analysis model used in this work. Section III provides more details about the EM model and calibration. In Section IV the details of the EM analysis in spintronic memory array is given. Section $\mathrm{V}$ presents the results of EM analysis and finally, Section VI concludes the paper.

\section{BACKGROUND}

\section{A. Spintronic Memory Technology}

The spintronic-based memory is an emerging non-volatile memory technology where the spin of the electron is utilized to store the data instead of the charge. The two most promising types of spintronic memory technology are the Spin Transfer Torque Magnetic Random Access Memory (STT-MRAM) and the Spin Orbit Torque Magnetic Random Access Memory (SOT-MRAM) [13]. These memories have high read/write current densities as compared to conventional CMOS memory technologies such as the Static Random Access Memory (SRAM) and hence are more susceptible to EM effects. In this work, we focus on EM analysis in the STT-MRAM technology since it is more mature as shown by several recent industrial demonstrations [14-17].

The Magnetic Tunnel Junction (MTJ) is the storage element in STT-MRAM technology, which stores the data in the form of resistance states. The MTJ consists of two ferromagnetic layers (e.g., $\mathrm{CoFeB}$ ) separated by a thin barrier oxide layer (e.g., $\mathrm{MgO}$ ) (see Fig. 1(a)). The magnetic orientation of one of the layers is fixed and is called the Reference Layer (RL). The magnetic orientation of the other layer, also called the Free Layer (FL), can be freely rotated. When the magnetic orientations of the two layers are parallel to each other ('P'configuration), the MTJ is in a low resistance state. On the other hand, when the magnetic orientations of FL and RL are anti-parallel ('AP' configuration), the MTJ has a high resistance. To switch the magnetic orientations of the MTJ from one configuration to the other, a write current is passed through the MTJ in the proper direction as given in Fig. 1(a) To read the content of the MTJ, a small read current is passed through the MTJ and the resistance state is sensed using a sense amplifier.

A typical STT-MRAM bit-cell structure is shown in Fig. 1(b) and is known as 1T-1MTJ (one transistor and one MTJ). The bit-cell has a total of three terminals, namely the source-line (SL), the bit-line (BL) and the word-line (WL). A typical STT-MRAM memory array organization is presented in Fig. 2(a) It consists of the 1T-1MTJ bit-cell as well as the peripheral circuitry, which are CMOS-based.

\section{B. Architecture and Mapping}

From an architectural point of view, a cache implementation can be seen as a hierarchy of different elements, each of them containing a mix of memory and control parts. The hierarchy breakdown of a typical STT-based second level cache is represented in Fig. 2(b) The amount of elements banks, instances and arrays - may vary from one architecture to another. In this example, as a common design choice, each array is composed of four sub-arrays arranged in a butterfly configuration, with the I/O and control logic placed between the memory areas.

In the experimental part of this work, the EM analysis is performed on a simulated system having an STT-MRAM second / last level cache integrated in a general purpose ARM $\mathrm{CPU}$ (more on this in Section V). The target cache is $512 \mathrm{kB}$ large and is designed to have 4 banks, 4 arrays per bank and 4 sub-arrays per array, adopting the aforementioned butterfly configuration. Due to the relatively small size, in this case the banks breakdown to instances is superfluous, i.e. there is a single instance corresponding to the bank itself.

To identify each word within the target cache, a special internal 16-bit address is used. This address is generated using a subset of the original 64-bit memory address, with additional way bits coming from the tag comparison logic (the cache is set-associative). Each part of the internal address is linked to 


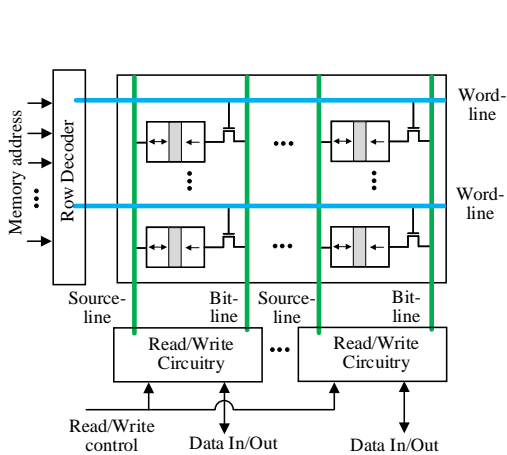

(a) STT-MRAM memory array

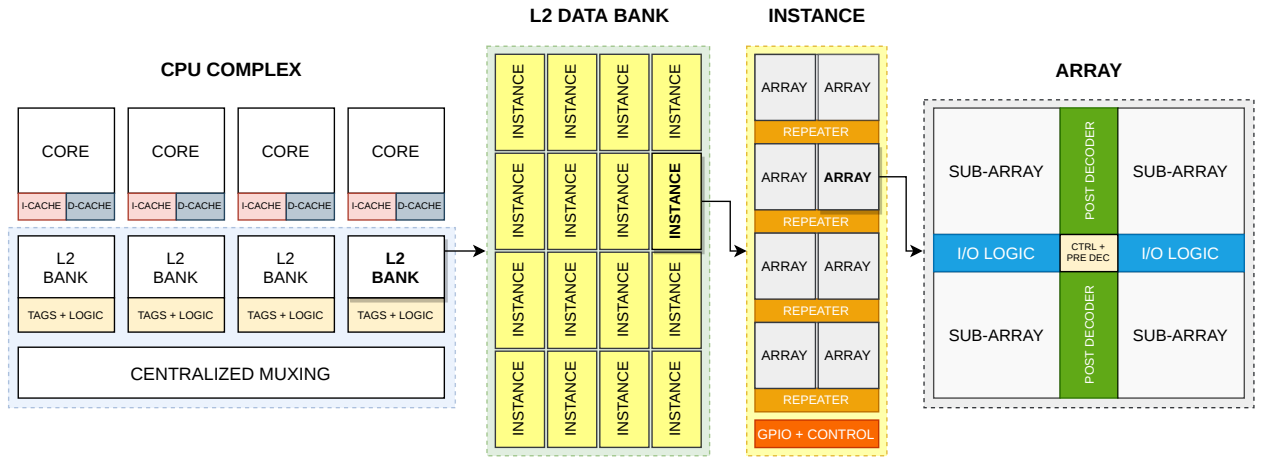

(b) STT-MRAM L2 cache architectural breakdown

Fig. 2: STT-MRAM memory and cache organization

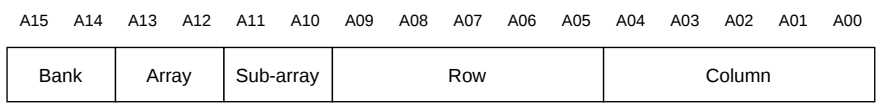

Fig. 3: Internal address for word selection in the target L2 cache

a specific physical element of the cache as shown in Fig. 3 . After the selection of the sub-array, 5 bits are left for the row (single WL) and 5 bits for the column (64 BLs, i.e. a word). The most relevant part for the context of this paper is the 5-bit row selection. Lowest values of the row address correspond to WLs closer to the sense amplifiers and vice versa.

During a read operation, after a WL is activated, all the bits contained in the corresponding memory cells are sensed from the BLs and transferred to the row buffer. Integrated multiplexers are then used to select a single word out of the content of the buffer. That is realized based on the above mentioned 5-bit column selection address. Since the L2 cache operates with blocks of 64 bytes, 8 words need to be read to assemble the full information, but the STT data array is only accessed once and subsequent reads are served by the row buffer. In case following requests target blocks belonging to the same row, they can be entirely satisfied by the row buffer.

\section{EM Modeling}

Most of the existing EM analysis methodologies use the Black's equation [18] to model the MTTF $\left(t_{50 \%}\right)$ due to EM. The Black's equation is given as:

$$
t_{50 \%}=\frac{A}{j^{n}} \cdot e^{\frac{E a}{k T}},
$$

where $E_{a}$ is the EM activation energy, $k$ is the Boltzmann constant, $T$ is the temperature in Kelvin, $j$ is the current density, $n$ is a constant (between 1 and 2) and A is a fitting parameter. The Black's model is an empirical model with only a few parameters. It does not consider the impact of residual stress and other material parameters affecting EM [19].

Most of the recent EM models have been developed from the solution of Korhonen's equation [20]. The hydrostatic stress evolution in space $(x)$ and time $(t), \sigma(x, t)$ along an interconnect line due to electromigration is modeled in Korhonen's equation as

$$
\frac{\partial \sigma}{\partial t}=\frac{\partial}{\partial x}\left[\kappa\left(\frac{\partial \sigma}{\partial x}+\frac{e Z \rho j}{\Omega}\right)\right] .
$$

Here, $e$ is the electron charge, $Z$ is the effective valence charge, $\rho$ is the resistivity of the metal line and $\kappa=D_{a} B \Omega / k T$, where $B$ is the effective bulk elasticity modulus, $\Omega$ is the atomic lattice volume and $D_{a}$ is the atomic diffusivity. The relation between the diffusion constant $\left(D_{0}\right)$ and activation energy $\left(E_{a}\right)$ is given by the Arrhenius equation as:

$$
D_{a}=D_{0} \cdot e^{-\frac{E_{a}}{k T}} .
$$

The existing works on EM analysis in the signal lines of SRAM [9, 10] are based on Blech length formulation, which is too simplistic. They do not consider the impact of various material parameters given in the Korhonen's equation on the EM failure times. In contrast, our EM analysis is based on a physics-based EM model derived form the solution of Korhonen's equation, as explained in the following section.

\section{Proposed EM Analysis Model}

\section{A. EM Model Calibration}

In our previous works [11, 12], we have proposed an EM model based on the solution of Korhonen's equation, which has only physical parameters. In this model, the time to failure (TTF) due to EM is divided into the following two phases.

1) Nucleation phase: This phase is characterized by a tensile stress build up due to the migration of atoms at the cathode end of the line close to the via bottom. This stress build up can be modeled as [21].

$\sigma=\sigma_{r e s}+\frac{e Z \rho j l}{\Omega}\left(\frac{1}{2}-\frac{x}{l}-4 \sum_{n=0}^{\infty} \frac{\cos \left(\frac{(2 n+1) \pi x}{l}\right)}{(2 n+1)^{2} \pi^{2}} e^{-\kappa \frac{(2 n+1)^{2} \pi^{2}}{l^{2}} t}\right)$

Here, $l$ is the length of the metal line, and $\sigma_{\text {res }}$ is the residual stress which is induced due to cooling down of processes happening at higher temperatures to room temperature as a result of mismatches in coefficients of thermal expansion between different materials. 
The void formation happens when the stress builds up and reaches a critical stress $\left(\sigma_{\text {crit }}\right)$. The instant in time when the stress at the cathode end of the line $(\sigma)$ reaches $\sigma_{\text {crit }}$ is defined as the nucleation time $\left(t_{n u c}\right)$. The resistance of the line does not increase during this phase.

2) Growth phase: The resistance of the line does not change significantly even after void nucleation. The void grows and reaches a critical size to cover the cross-section of the line, after which the resistance of the line starts to increase [22]. The time required for the void to grow to a critical size $\left(\triangle l_{\text {crit }}\right)$ after $\sigma_{\text {crit }}$ is reached is called the incubation time $\left(t_{i}\right)$. It can be modeled as [23]:

$$
t_{i}=\frac{\triangle l_{\text {crit }} \cdot k T}{D_{a} e Z \rho j} .
$$

A sharp increase in resistance is observed once the void grows to a critical size, A gradual resistance increase is observed after this due to the current shunting by liner barriers [24-26]. The increase of wire resistance in this phase $(\Delta r(t))$ can be approximated as:

$$
\triangle r(t)=\frac{D_{a}}{k T} \cdot e Z \rho_{c u} j \cdot\left[\frac{\rho_{T a}}{h_{T a}(2 H+W)}-\frac{\rho_{C u}}{H W}\right] .
$$

Here, $\rho_{T a}$ and $\rho_{C u}$ are the resistivities of the barrier material ( $\mathrm{Ta} / \mathrm{TaN}$ ) and $\mathrm{Cu}$ respectively, $W$ is the line width, $H$ is the $\mathrm{Cu}$ thickness and $h_{T a}$ is the barrier layer thickness.

This EM model was calibrated for a metal line of width $23 \mathrm{~nm}$ and height $60 \mathrm{~nm}$ for a current density of $1.68 \mathrm{MA} / \mathrm{cm}^{2}$ in our previous works [11, 12]. The measurements were done at accelerated temperatures of $230^{\circ} \mathrm{C}, 280^{\circ} \mathrm{C}$ and $330^{\circ} \mathrm{C}$. The tests were obtained at package level with a continuous monitor of the resistance versus time which allows an accurate estimation of the failure times. A $20 \%$ resistance increase was used as the failure criterion.

The next step was to get the various parameters of the EM model from the measurement data. $E_{a}$ was derived using Black's equation by conducting EM experiments at different temperatures under constant current density. The values of $\mathrm{Z}, \sigma_{\text {res }}$ and $\sigma_{\text {crit }}$ were obtained from [27]. The value of $\mathrm{D}_{0}$ was then derived using the minimum Root Mean Square Error (RMSE) method, which minimizes the error between the modeled and measured TTFs at different temperatures. The reader can refer to our previous works ([11, 12]) for a detailed description of the methodology followed.

However, for the bit-lines of the STT array, the dimensions as well as the current density are different from those in [11, 12]. The width is $32 \mathrm{~nm}$ and the height is $64 \mathrm{~nm}$. The current density depends on the memory operation to be performed, whether it is a read operation or a write operation. Hence the EM model has to be scaled based on the new dimensions of the BL as explained below.

\section{B. Scaling the EM Model Parameters}

This section explains how the EM model parameters are scaled based on the dimensions of the BL and is based on the work published in [28].
1) Effective Diffusivity $\left(D_{0}\right)$ : The value of $D_{0}$ at $23 \mathrm{~nm}$ is obtained as $1.9 \times 10^{-9}$ in [12]. As explained in [28], as a first approach, a power trend-line can be fit to the data in [28] to obtain the scaled value of $D_{0}$ at $32 \mathrm{~nm}$. Based on this approach, the value of $D_{0}$ at $32 \mathrm{~nm}$ can be approximated as $1.9 \times 10^{-9} / 2.47=7.7 \times 10^{-10} \mathrm{~m}^{2} \mathrm{~s}^{-1}$.

2) Effective elastic modulus/confinement $(B)$ : From the data in [28], we see that the B values for $23 \mathrm{~nm}$ and $32 \mathrm{~nm}$ are very similar. Hence, we use the same value of B at $32 \mathrm{~nm}$ as that at $23 \mathrm{~nm}$, which is $13.4 \mathrm{GPa}$.

3) Line Resistivity $(\rho)$ : Based on the trend shown in [28], the $\rho$ value of $57 \Omega \cdot \mathrm{nm}$ at $23 \mathrm{~nm}$ is scaled to $\rho=43.7 \Omega \cdot \mathrm{nm}$ at $32 \mathrm{~nm}$.

4) Residual Stress $\left(\sigma_{\text {res }}\right)$ : It can be shown that $\sigma_{\text {res }}$ follows a trend consistent with the effective bulk modulus (B) variations [28]. Since B does not change from $23 \mathrm{~nm}$ to $32 \mathrm{~nm}, \sigma_{\text {res }}$ is also taken to be same at $32 \mathrm{~nm}$.

5) Critical Stress $\left(\sigma_{\text {crit }}\right)$ : It can be shown that the critical stress

$$
\sigma_{\text {crit }}=\sqrt{k \frac{G_{c} B}{h}}
$$

where, $G_{c}$ is the interface energy (per unit area), $h$ is the line height and $k$ is an empirical calibration factor. Here, $G_{c}, B$ and $k$ can be assumed to be constants, hence $\sigma_{\text {crit }} \propto \sqrt{\frac{1}{h}}$. This means that $\sigma_{\text {crit }} @ 64 \mathrm{~nm}=\sqrt{\frac{60}{64}} \cdot \sigma_{\text {crit }} @ 60 \mathrm{~nm}$.

The only temperature-dependent parameters are residual and critical stress. By extrapolation, the $\sigma_{\text {res }}$ and $\sigma_{\text {crit }}$ at $100^{\circ} \mathrm{C}$ and $60 \mathrm{~nm}$ wire height are 189.95 and $242.85 \mathrm{MPa}$ respectively.

\section{EM ANALYSIS IN STT-MRAM ARRAY}

The circuit simulations of STT-MRAM show the STTMRAM read and write latencies in the typical case for a $256 \mathrm{~KB}$ data array is around $3.9 \mathrm{~ns}$ and $9 \mathrm{~ns}$ [29]. The typical read current $\left(I_{r}\right)$ for STT is around $50 \mu \mathrm{A}$. This read current is roughly aligned with [29]. The write current depends on the switching direction. The typical write current for 'AP' $\rightarrow$ ' $\mathrm{P}$ ' (' 1 ' $\rightarrow$ ' 0 ') switching $\left(I_{w 0}\right)$ is around $70 \mu \mathrm{A}$, whereas the write current for ' $\mathrm{P}$ ' $\rightarrow$ 'AP' ('0' $\rightarrow$ ' 1 ') switching $\left(I_{w 1}\right)$ is around $120 \mu \mathrm{A}$. These current ranges are in agreement with other magnetic STT process design kits (PDK) like [30]. The read current shares one of the write current paths. $I_{r}$ is typically designed to be in the 'P' $\rightarrow$ 'AP' switching direction to minimize the probability of read-disturb [31, 32]. Hence, the average current through the bit-line $\left(I_{B L}\right)$ can be computed as:

$$
I_{B L}=\left(I_{w 1}+I_{r}-I_{w 0}\right) / 3
$$

Based on the above values, $I_{B L}$ can be computed to be $33.33 \mu \mathrm{A}$, which corresponds to a current density of of 1.63 $\mathrm{MA} / \mathrm{cm}^{2}$. Hence, the EM analysis has to be done at this current density. The above calculation is based on the assumption that the number of read, write ' 0 ' and write ' 1 ' operations are 


\begin{tabular}{|c|c|c|c|c|c|c|c|}
\hline \multirow{2}{*}{ Length $(\mu \mathrm{m})$} & \multicolumn{3}{|c|}{ Failure Time (hours) at $230^{\circ} \mathrm{C}$} & \multirow{2}{*}{ Length $(\mu \mathrm{m})$} & \multicolumn{3}{|c|}{ Failure Time (years) at $100^{\circ} \mathrm{C}$} \\
\hline & Nucleation & Growth & Total & & Nucleation & Growth & Total \\
\hline$\leq 12.6$ & NA & - & - & $\leq 20.3$ & NA & 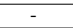 & - \\
\hline 12.7 & 308.33 & 195.42 & 503.75 & 20.4 & 10.03 & 18.57 & 28.6 \\
\hline 13 & 211.11 & 195.46 & 406.67 & 21 & 9.97 & 18.59 & 28.56 \\
\hline 14 & 155.56 & 196.05 & 351.60 & 22 & 9.91 & 18.63 & 28.54 \\
\hline 15 & 138.89 & 196.53 & 335.42 & 23 & 9.87 & 18.67 & 28.54 \\
\hline 16 & 127.78 & 197.02 & 324.80 & 24 & 9.85 & 18.70 & 28.55 \\
\hline 17 & 125.00 & 197.51 & 322.51 & 25 & 9.83 & 18.74 & 28.57 \\
\hline 18 & 122.22 & 197.99 & 320.21 & 26 & 9.82 & 18.78 & 28.6 \\
\hline 19 & 119.44 & 198.48 & 317.92 & 27 & 9.81 & 18.82 & 28.63 \\
\hline 20 & 119.44 & 198.96 & 318.41 & 28 & 9.81 & 18.86 & 28.67 \\
\hline
\end{tabular}

TABLE I: EM failure times for different lengths at accelerated temperature of $230^{\circ} \mathrm{C}$ as well as operational temperature of $100^{\circ} \mathrm{C}$.

\begin{tabular}{lllllll}
\hline Level & Technology & Size & Assoc & Read & Write & Banks \\
\hline L1I & SRAM & $32 \mathrm{kB}$ & 2 & 4 & 4 & - \\
L1D & SRAM & $32 \mathrm{kB}$ & 4 & 4 & 4 & - \\
L2 & STT-MRAM & $512 \mathrm{kB}$ & 16 & 9 & 16 & 4 \\
\hline
\end{tabular}

TABLE II: Simulated cache subsystem specifications

equal. However, this assumption is not reasonable since the number of read/write operations heavily depends on the workload. Hence the average current through each bit-line, $I_{B L}$, has to be considered separately based on realistic workloads to determine the impact of EM. In this case, the Eq. 8 needs to be modified as given below:

$$
I_{B L}=\left(I_{w 1} \cdot n_{w 1}+I_{r} \cdot n_{r}-I_{w 0} \cdot n_{w 0}\right) / n_{t}
$$

Here, $n_{w 1}$ is the number of write ' 1 ' operations, $n_{w 0}$ is the number of write ' 0 ' operations, $n_{r}$ is the number of read operations and $n_{t}$ is the total number of operations (read plus write) in a BL estimated based on the workload.

The EM analysis in STT array is based on the scaled model parameters discussed in Section III-B. In addition, the impact of current density $(j)$ and the workload have a significant impact on the EM failure times.

The EM failure times of the BL for different metal line lengths are shown in Table 1 for an $I_{B L}$ of $33.33 \mu \mathrm{A}$. For the Cobalt (Co) cap technology at $25 \mathrm{~nm}$ linewidth, typically a

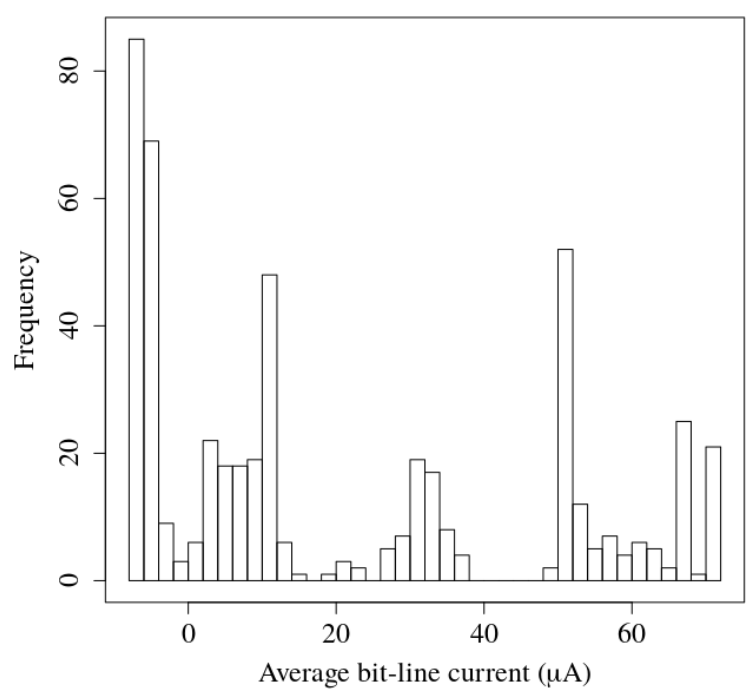

Fig. 4: Histogram of $I_{B L}$ for 'mcf' workload

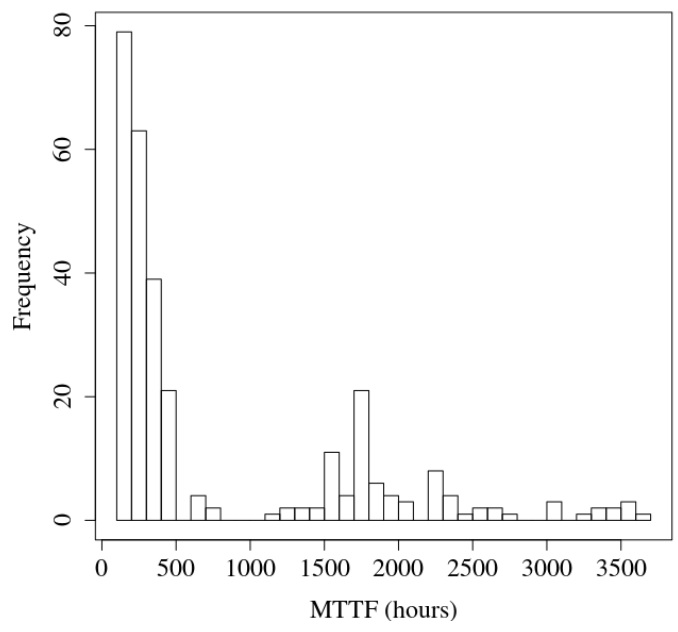

(a)

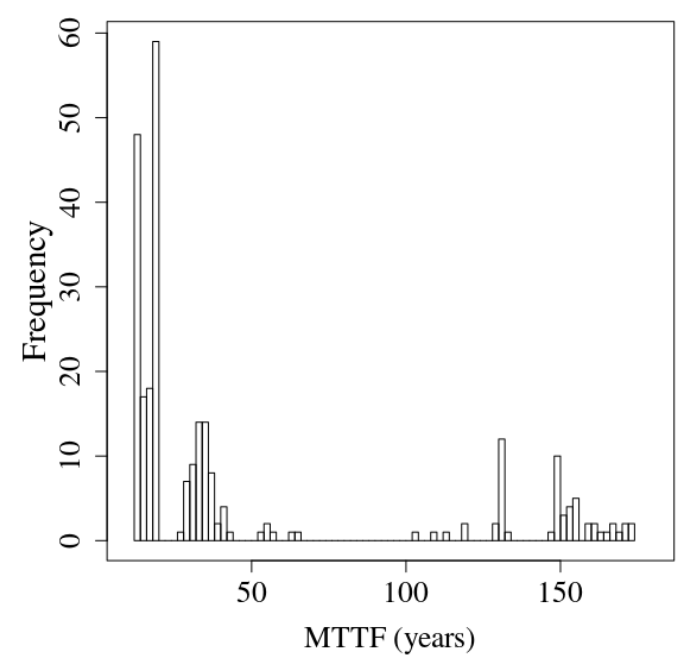

(b)

Fig. 5: Histogram of MTTF for 'mcf' workload, (a) at $230^{\circ} \mathrm{C}$ $($ Median $=327$ hours $),(b)$ at $100^{\circ} \mathrm{C}($ Median $=18.36$ years $)$

maximum DC current of $<100 \mu \mathrm{A}$ has been considered for $100 \mathrm{~K} \mathrm{POH}$ (power-on hours) at $100{ }^{\circ} \mathrm{C}$. According to [28], even for the Co cap, by scaling down the technology to $16 \mathrm{~nm}$ (1.56x) the maximum current reduced for 4.14 times. This means that the maximum DC current for $100 \mathrm{~K} \mathrm{POH}$ at 100 ${ }^{\circ} \mathrm{C}$ in this technology is around $24 \mu \mathrm{A}$. The technology used in this work is dielectric cap technology with bare undoped copper and the maximum current in this technology is even less than $24 \mu \mathrm{A}$. The average current of $33.33 \mu \mathrm{A}$ is obviously larger than the maximum tolerable current in this technology and temperature, hence, considering the workload-dependent EM risk is essential.

At $230^{\circ} \mathrm{C}$ and $100^{\circ} \mathrm{C}$, no EM failure is observed for a length $\leq 12.6 \mu \mathrm{m}$ and $\leq 20.3 \mu \mathrm{m}$ respectively. This is because no void nucleation occurs below this length. This critical length $\left(L_{c}\right)$ is called the Blech length. For copper, $j \times L_{c}$ can vary from $2000 \mathrm{~A} / \mathrm{cm}$ to around $5000 \mathrm{~A} / \mathrm{cm}$. The value of $L_{c}$ based on our model at $230^{\circ} \mathrm{C}$ and $100^{\circ} \mathrm{C}$ are $12.6 \mu \mathrm{m}$ and $20.3 \mu \mathrm{m}$ 


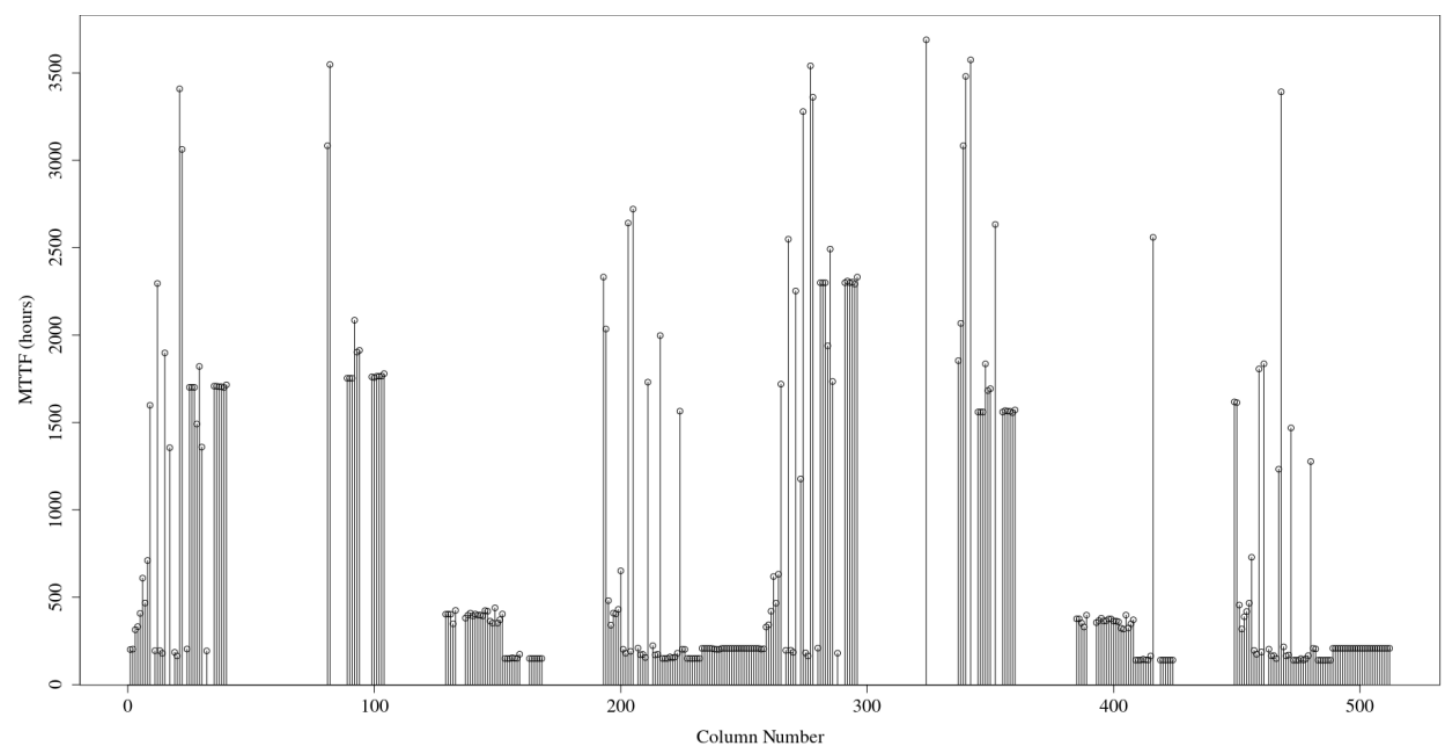

(a)

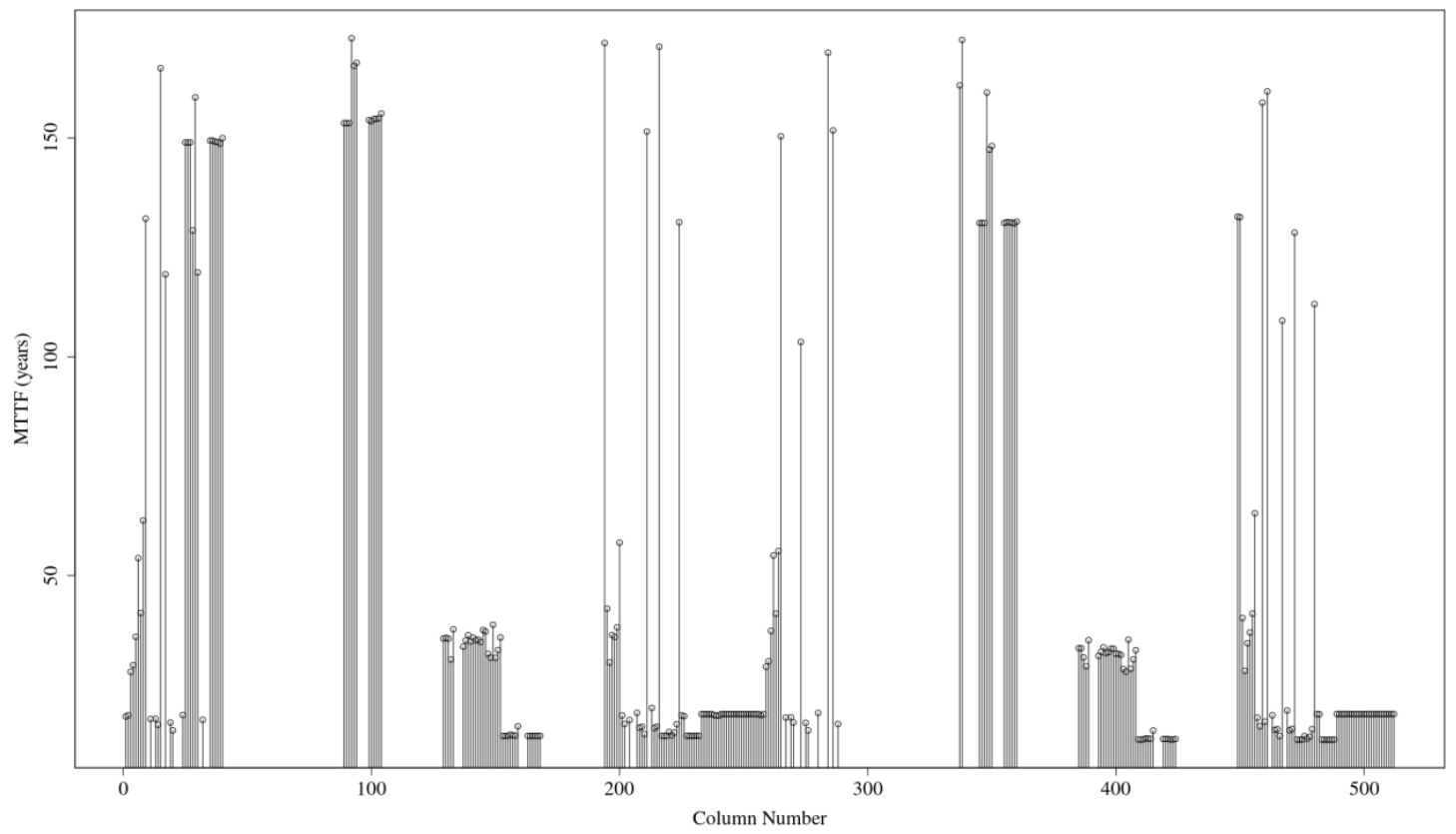

(b)

Fig. 6: Column-wise MTTF for 'mcf' $(0=\mathrm{MSB} ; 512=\mathrm{LSB})$, The columns with no values are immortal to EM failures. (a) at $230{ }^{\circ} \mathrm{C}$ (number of immortal columns: 218 ) and (b) at $100^{\circ} \mathrm{C}$ (number of immortal columns: 248)

according to Table I This corresponds to a $j \times L_{c}$ value of $2050 \mathrm{~A} / \mathrm{cm}$ at $230^{\circ} \mathrm{C}$ and $3303.7 \mathrm{~A} / \mathrm{cm}$ at $100^{\circ} \mathrm{C}$. From Table $]$ it can be seen that the nucleation time saturates after a certain length, as expected. This length is around $19 \mu \mathrm{m}$ at $230^{\circ} \mathrm{C}$ and $27 \mu \mathrm{m}$ at $100^{\circ} \mathrm{C}$ as given in the table.

The typical STT array configurations are $64 \times 64,128 \times 128$, $256 \times 256$ and $512 \times 512$ bit-cells. We estimate the bit-cell height/width to be around $200 \mathrm{~nm}$. Based on this, the BL lengths vary from a minimum of $12.8 \mu \mathrm{m}$ to a maximum of $102.4 \mu \mathrm{m}$. Except for the $64 \times 64$ configuration at $100^{\circ} \mathrm{C}$, these lengths are clearly much greater than $L_{c}$ and hence these bitlines can likely be subjected to EM failures.

\section{RESULts}

\section{A. Simulation Setup}

The number of read and write operations in the memory array, as well as the data values being accessed, heavily depend on the workload. Hence, it was chosen to model this dependency for a CPU using an in-order ARMv8-A core, separate L1 instruction and data caches and unified L2 cache. This 


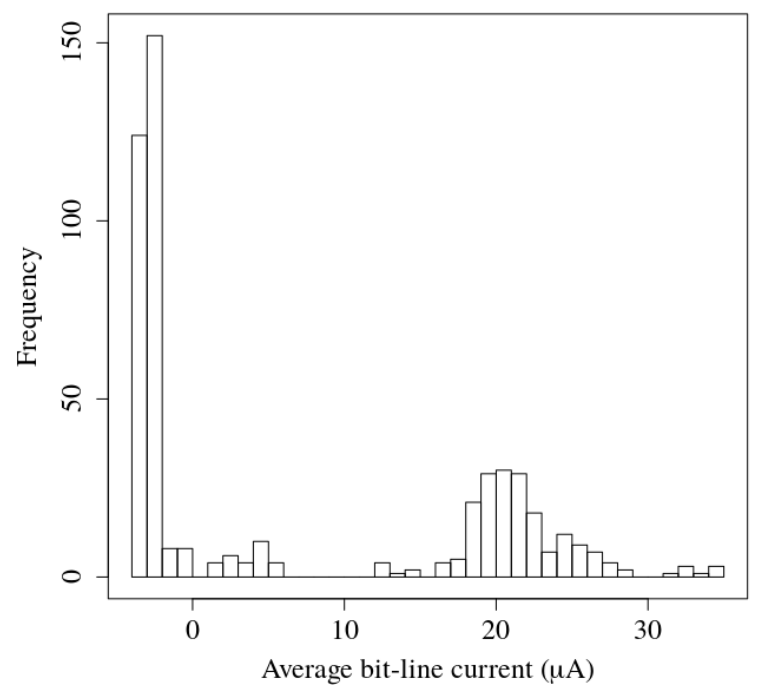

Fig. 7: Histogram of $I_{B L}$ for 'gcc' workload

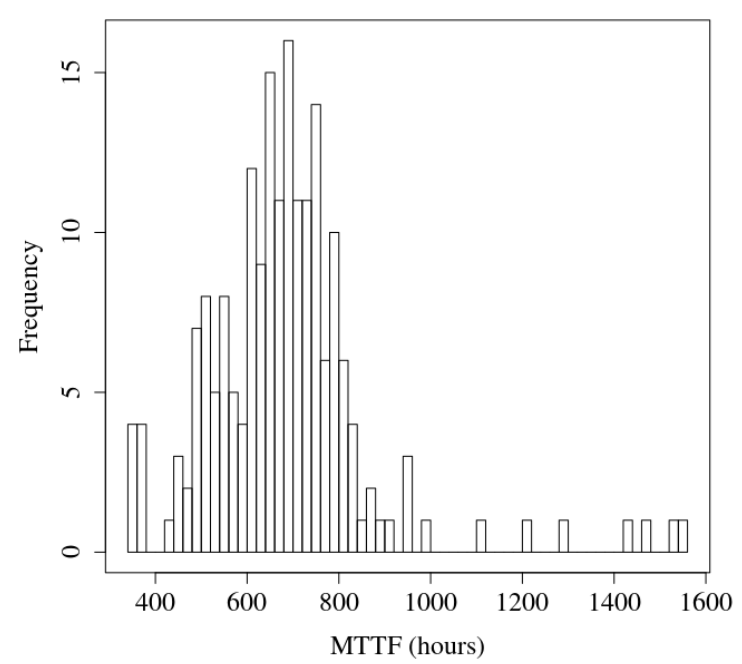

(a)

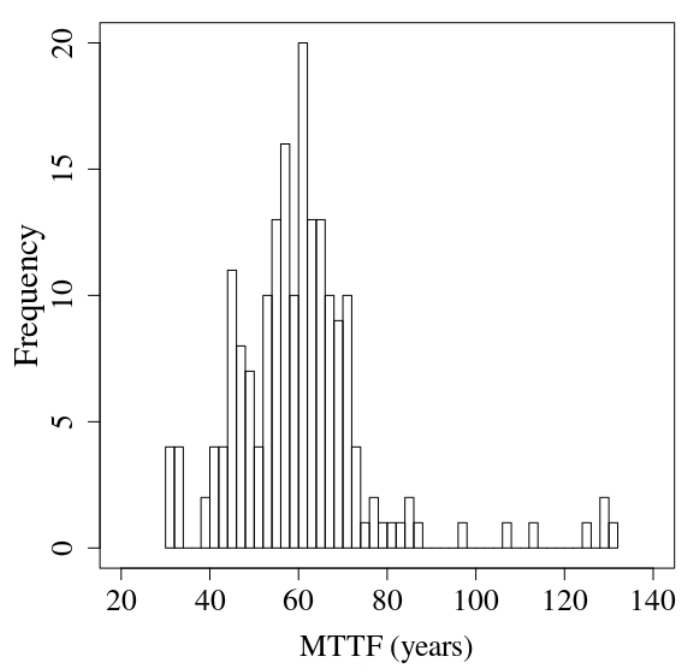

(b)

Fig. 8: Histogram of MTTF for 'gcc' workload, (a) at $230^{\circ} \mathrm{C}$ (Median $=674$ hours $),(\mathrm{b})$ at $100^{\circ} \mathrm{C}($ Median $=59.46$ years $)$ system was modelled in a gem5-based simulator framework optimized for non-volatile memory exploration [29, 33].

The specifications of the cache subsystem for the simulated platform can be found in Table III Read and write latencies are expressed in cycles. Banking contention was ignored for the first level cache since it is not relevant for this work. The cache line, which represents the unit of communication within the cache subsystem, has a fixed size of 64 bytes. Both the L1 and L2 caches are mostly-inclusive. Additional details about the layout of the modeled L2 cache, which are needed after the simulation phase to perform the EM analysis, are described in Section II]

Two workloads from the integer SPEC CPU(C) 2017 benchmark suite were used to stress the L2 cache, namely 602.gcc_s and 607.mcf_s. The 'gcc' workload is a $\mathrm{C}$ compiler and its performance heavily depends on cache latency and size [34], while 'mcf' is a public transportation scheduling application and has a very large memory footprint [35]. For the sake of completeness, the benchmarks were executed using the train input dataset and a single chunk of 100 million instructions was selected for each of them to reduce simulation times.

While running these applications inside the simulator, L2 cache access traces were extracted gathering addresses and data from packets coming from upper cache levels (e.g. L1I/D misses or L1D writebacks) and the main memory (e.g. prefetches or write fills after L2 misses). The binary values served as input to perform the workload dependent EM analysis on the respective signal lines of the cache macro. As a side note, the traces contained a limited amount of duplicated access entries, in the cases a request got rejected because a bank was busy. Due to the nature and quantity of the requests, this redundancy should have no impact on the conclusions of our work.

\section{B. Results}

As explained in Section IV] the actual $I_{B L}$ in each bit-line depends on the workload. Hence, we investigate the impact of the aforementioned workloads on the MTTF of each BL in a $512 \times 512$ array. As an illustration, we show the MTTF values at an accelerated temperature of $230^{\circ} \mathrm{C}$ as well as operational temperature of $100^{\circ} \mathrm{C}$. The same trend can be seen at other temperatures as well, with corresponding changes in the absolute MTTF values.

The histogram of $I_{B L}$ and the resulting MTTF for the 'mcf' workload is given in Fig. 4 and Fig. 5, respectively. At the accelerated temperature of $230^{\circ} \mathrm{C}$, the MTTF values are comparatively smaller than $100^{\circ} \mathrm{C}$. From the figures, it can be seen that there is significant variation in the $I_{B L}$ and MTTF in different bit-lines of the STT array. Furthermore, some of the bit-lines are immortal to EM failures due to the low $I_{B L}$ in these BLs. This is illustrated in Fig. 6, which shows the MTTF in each of the 512 columns. In particular, the variables in the algorithms that are executed to produce the workload have more significant bits which rarely change leading to much lower switching activity compared to the less significant bits. These variables are assigned to the memory 


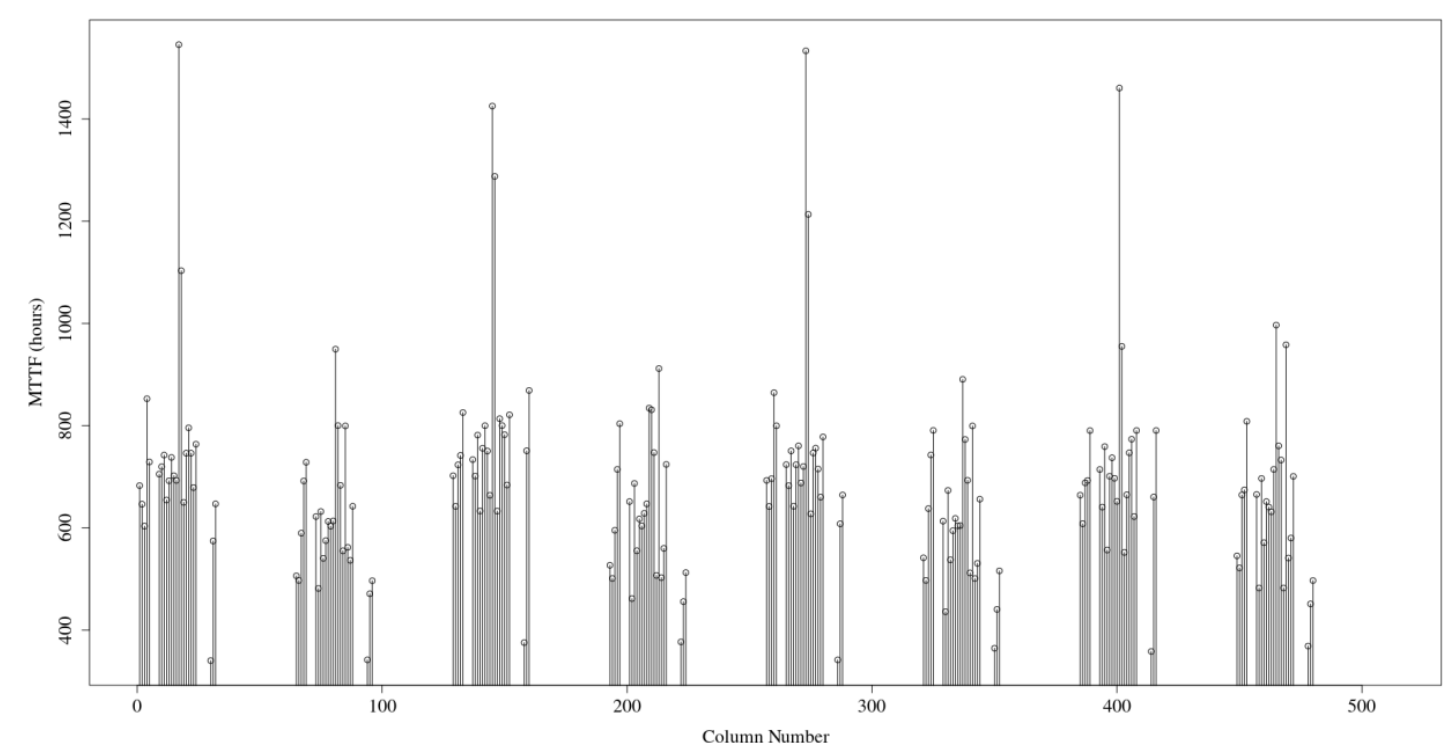

(a)

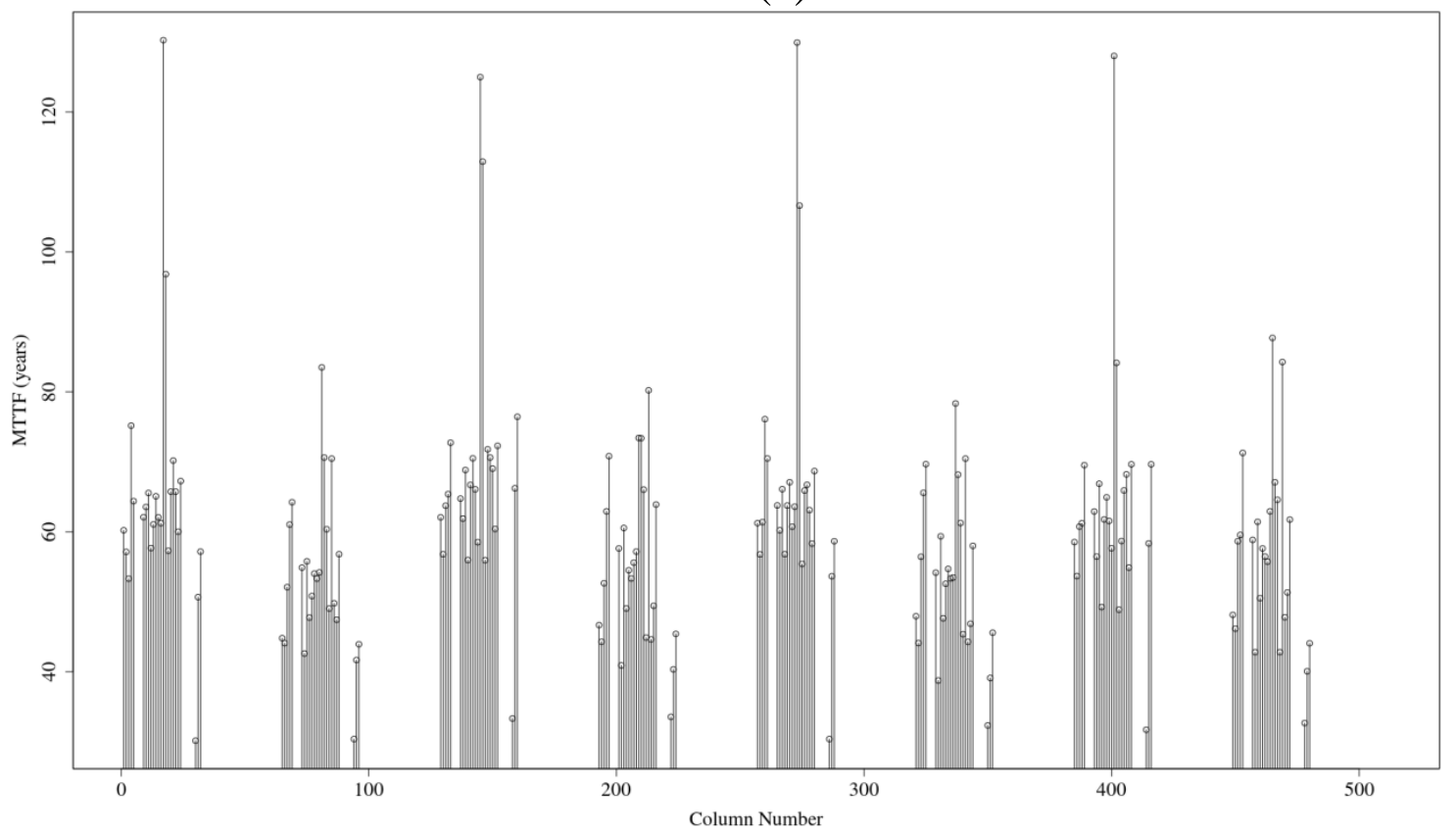

(b)

Fig. 9: Column-wise MTTF for 'gcc' $(0=\mathrm{MSB} ; 512=\mathrm{LSB})$, The columns with no values are immortal to EM failures. (a) at $230^{\circ} \mathrm{C}$ (number of immortal columns: 320 ) and (b) at $100^{\circ} \mathrm{C}$ (number of immortal columns: 320 )

layouts in an interleaved way, leading to peaks and valleys of EM stress. The figure shows that in addition to the significant variation of MTTF between the columns, at $230^{\circ} \mathrm{C}$ and $100^{\circ} \mathrm{C}$ a total of 218 and 248 columns are immortal to EM failures respectively.

Fig. 7. Fig. 8 and Fig. 9 show the EM analysis for the 'gcc' workload. The trends shown by this workload are similar to those of the 'mcf' workload. However, the $I_{B L}$ for ' $\mathrm{gcc}$ ' is lower than that of 'mcf', which lead to higher median values of MTTF for the 'gcc' workload. This also results in more EM immortal bit-lines for the 'gcc' workload. This is presented in Fig. 9, where the total number of immortal BLs for ' $\mathrm{gcc}$ ' at both $230^{\circ} \mathrm{C}$ and $100^{\circ} \mathrm{C}$ is 320 as compared to 218 and 248 for the 'mcf' workload at $230^{\circ} \mathrm{C}$ and $100^{\circ} \mathrm{C}$ respectively. This shows that large variations are not only present inside one workload but also across different workloads. This comparative analysis highlights the importance of performing workload-dependent EM analysis to obtain realistic EM failure times and hence incorporate proper EM mitigation techniques for the STT-MRAM array. This is a strong motivation for our 
extended physics-based model as proposed in this paper.

The overall MTTF of the memory is determined by the smallest MTTF of the wires. In these workloads, the maximum average current is $71.13 \mu \mathrm{A}$ which happens at the bit-line number 484 under the 'mcf' workload. At $100^{\circ} \mathrm{C}$ the lifetime of this interconnect is 12.47 years. To put this number into perspective, we consider $5 \%$ process variation for the width and thickness of the wire as well as the current. As a result, lifetime of this wire can be reduced to 9.2 years, corresponding to $26.2 \%$ reduction in the overall lifetime.

\section{CONCLUSIONS}

In this work, we performed electromigration analysis on the signal lines (BL) of STT-MRAM memory array based on realistic workloads. The analysis was done with the help of recently developed physics-based EM model, which is calibrated based on measurement data and scaled to fit the dimensions and material parameters of the STT array. The results show that EM can be a potential reliability issue in the signal lines of STT array mainly due to the increased current densities. This work also highlights the importance of considering workload dependencies while performing EM analysis and incorporating the required mitigation techniques.

\section{REFERENCES}

[1] J. P. Gambino, T. C. Lee, F. Chen, and T. D. Sullivan, "Reliability challenges for advanced copper interconnects: Electromigration and time-dependent dielectric breakdown (TDDB)," in $16^{\text {th }}$ IEEE International Symposium on the Physical and Failure Analysis of Integrated Circuits, pp. 677-684, 2009.

[2] E. T. Ogawa, K.-D. Lee, V. A. Blaschke, and P. S. Ho, "Electromigration reliability issues in dual-damascene $\mathrm{Cu}$ interconnections," IEEE Transactions on reliability, vol. 51, no. 4, pp. 403-419, 2002.

[3] C. M. Tan and A. Roy, "Electromigration in ULSI interconnects," Materials Science and Engineering: R: Reports, vol. 58, no. 1-2, pp. 1-75, 2007.

[4] A. D. Kent and D. C. Worledge, "A new spin on magnetic memories," Nature nanotechnology, vol. 10, no. 3, p. 187, 2015.

[5] H.-S. P. Wong and S. Salahuddin, "Memory leads the way to better computing," Nature nanotechnology, vol. 10, no. 3, p. 191, 2015.

[6] X. Huang, Y. Tan, V. Sukharev, and S. X.-D. Tan, "Physics-based electromigration assessment for power grid networks," in Design Automation Conference (DAC), pp. 1-6, 2014.

[7] H. Zhou, S. Yu, Z. Sun, and S. X.-D. Tan, "Reliable Power Grid Network Design Framework Considering EM Immortalities for Multi-Segment Wires," in Asia and South Pacific Design Automation Conference (ASPDAC), pp. 74-79, 2020.

[8] S. Ma, X. Wang, S. X.-D. Tan, L. Chen, and J. He, "An Adaptive Electromigration Assessment Algorithm for Full-chip Power/Ground Networks," in Asia and
South Pacific Design Automation Conference (ASPDAC), pp. 38-43, 2020.

[9] Z. Guan, M. Marek-Sadowska, and S. Nassif, "SRAM bit-line electromigration mechanism and its prevention scheme," in International Symposium on Quality Electronic Design (ISQED), pp. 286-293, 2013.

[10] Z. Guan, M. Marek-Sadowska, and S. Nassif, "Statistical analysis of process variation induced SRAM electromigration degradation," in International Symposium on Quality Electronic Design (ISQED), pp. 700-707, 2014.

[11] S. M. Nair, R. Bishnoi, M. B. Tahoori, H. Zahedmanesh, K. Croes, K. Garello, G. S. Kar, and F. Catthoor, "Variation-Aware Physics-Based Electromigration Modeling and Experimental Calibration for VLSI Interconnects," in 2019 IEEE International Reliability Physics Symposium (IRPS), pp. 1-6, 2019.

[12] S. M. Nair, R. Bishnoi, M. B. Tahoori, H. Zahedmanesh, K. Croes, K. Garello, G. S. Kar, and F. Catthoor, "Physics based modeling of bimodal electromigration failure distributions and variation analysis for VLSI interconnects," in 2020 IEEE International Reliability Physics Symposium (IRPS), pp. 1-5, 2020.

[13] F. Oboril, R. Bishnoi, M. Ebrahimi, and M. B. Tahoori, "Evaluation of hybrid memory technologies using SOTMRAM for on-chip cache hierarchy," IEEE Transactions on Computer-Aided Design of Integrated Circuits and Systems, vol. 34, no. 3, pp. 367-380, 2015.

[14] K. Lee et al., "1Gbit High Density Embedded STTMRAM in 28nm FDSOI Technology," in 2019 IEEE International Electron Devices Meeting (IEDM), pp. 2-2, 2019.

[15] J. Alzate et al., "2 MB Array-Level Demonstration of STT-MRAM Process and Performance Towards L4 Cache Applications," in 2019 IEEE International Electron Devices Meeting (IEDM), pp. 2-4, 2019.

[16] J.-H. Park et al., "A novel integration of STT-MRAM for on-chip hybrid memory by utilizing non-volatility modulation," in 2019 IEEE International Electron Devices Meeting (IEDM), pp. 2-5, 2019.

[17] G. Hu et al., "Spin-transfer torque MRAM with reliable 2 ns writing for last level cache applications," in 2019 IEEE International Electron Devices Meeting (IEDM), pp. 2-6, 2019.

[18] J. R. Black, "Electromigration-A brief survey and some recent results," IEEE Transactions on Electron Devices, vol. 16, no. 4, pp. 338-347, 1969.

[19] V. Sukharev, "Beyond Black's equation: Full-chip EM/SM assessment in 3D IC stack," Microelectronic Engineering, vol. 120, pp. 99-105, 2014.

[20] M. Korhonen, P. Bo/rgesen, K. Tu, and C.-Y. Li, "Stress evolution due to electromigration in confined metal lines," Journal of Applied Physics, vol. 73, no. 8, pp. 3790-3799, 1993.

[21] X. Huang, V. Sukharev, T. Kim, H. Chen, and S. X.-D. Tan, "Electromigration recovery modeling and analysis under time-dependent current and temperature stressing," 
in $21^{\text {st }}$ Asia and South Pacific Design Automation Conference (ASP-DAC), pp. 244-249, 2016.

[22] K. Croes, M. Lofrano, C. Wilson, L. Carbonell, Y. K. Siew, G. Beyer, and Z. Tôkei, "Study of void formation kinetics in $\mathrm{Cu}$ interconnects using local sense structures," in 2011 IEEE International Reliability Physics Symposium (IRPS), pp. 3E-5, 2011.

[23] S. X.-D. Tan, H. Amrouch, T. Kim, Z. Sun, C. Cook, and J. Henkel, "Recent advances in EM and BTI induced reliability modeling, analysis and optimization," Integration, vol. 60, pp. 132-152, 2018.

[24] J. Michelon, C. Bruynseraede, D. Tio Castro, P. Roussel, R. Hoofman, and K. Maex, "Electromigration study of sub-100nm Cu-lines," in Advanced Metallization Conference (AMC), pp. 253-257, 2004.

[25] K.-D. Lee, Y.-J. Park, and B. Hunter, "The impact of partially scaled metal barrier shunting on failure criteria for copper electromigration resistance increase in $65 \mathrm{~nm}$ technology," in 2005 IEEE International Reliability Physics Symposium (IRPS), pp. 31-35, 2005.

[26] D. Tio Castro, R. Hoofman, J. Michelon, D. Gravesteijn, and C. Bruynseraede, "Void growth modeling upon electromigration stressing in narrow copper lines," Journal of Applied Physics, vol. 102, no. 12, p. 123515, 2007.

[27] H. Zahedmanesh, P. R. Besser, C. J. Wilson, and K. Croes, "Airgaps in nano-interconnects: Mechanics and impact on electromigration," Journal of Applied Physics, vol. 120, no. 9, p. 095103, 2016.

[28] H. Zahedmanesh et al., "Copper Electromigration; Prediction of Scaling Limits," in IITC, 2019.

[29] T. Evenblij, M. Perumkunnil, F. Catthoor, S. Sakhare, P. Debacker, G. Kar, A. Furnemont, N. Bueno, J. I. Gómez-Pérez, and C. Tenllado, "A Comparative Analysis on the Impact of Bank Contention in STT-MRAM and SRAM Based LLCs," in 37th International Conference on Computer Design (ICCD), pp. 255-263, 2019.

[30] F. Bernard-Granger, B. Dieny, R. Fascio, and K. Jabeur, "SPITT: A magnetic tunnel junction SPICE compact model for STT-MRAM," in Proceedings of the MOS-AK Workshop of the Design, Automation \& Test in Europe (DATE), 2015.

[31] R. Bishnoi, M. Ebrahimi, F. Oboril, and M. B. Tahoori, "Read disturb fault detection in STT-MRAM," in International Test Conference (ITC), pp. 1-7, 2014.

[32] S. M. Nair, R. Bishnoi, and M. B. Tahoori, "Mitigating Read Failures in STT-MRAM," in $38^{\text {th }}$ VLSI Test Symposium (VTS), pp. 1-6, 2020.

[33] N. Binkert, B. Beckmann, G. Black, S. K. Reinhardt, A. Saidi, A. Basu, J. Hestness, D. R. Hower, T. Krishna, S. Sardashti, R. Sen, K. Sewell, M. Shoaib, N. Vaish, M. D. Hill, and D. A. Wood, "The Gem5 Simulator," SIGARCH Comput. Archit. News, vol. 39, p. 1-7, Aug. 2011.

[34] SPECC Corporation, "602.gcc_s Benchmark Description." https://www.spec.org/cpu2017/Docs/benchmarks/ 602.gcc_s.html Accessed: 2020.
[35] SPEC(C) Corporation, "605.mcf_s Benchmark Description." https://www.spec.org/cpu2017/Docs/benchmarks/ 605.mcf_s.html Accessed: 2020. 